\title{
Identification of new regulatory genes controlling synthesis of folate-dependent enzymes in Aspergillus nidulans
}

\author{
Irmina Lewandowska, ${ }^{1}$ Małgorzata Balińska² and Andrzej Paszewski'
}

Author for correspondence: Andrzej Paszewski. Fax: +48 39121623.

e-mail: apasz@ibbrain.ibb.waw.pl

1 Institute of Biochemistry and Biophysics, Polish

Academy of Sciences, 5A Pawińskiego St, 02-106

Warszawa, Poland

2 Nencki Institute of Experimental Biology, Polish Academy of Sciences, 3 Pasteur St, 02 093 Warszawa, Poland

\begin{abstract}
Prototrophic revertants of a metH2 strain of Aspergillus nidulans which is impaired in the regulation of synthesis of folate-dependent enzymes were isolated and six of them analysed. In three of the isolates reversion was the result of an intragenic suppressor mutation in the metH locus. In the remaining strains suppressor mutations occurred in independent genes. These genes, designated folA, folB and folC, are linked and located in chromosome VI. Mutations in these genes render synthesis of some folate enzymes, particularly folylpolyglutamate synthetase, insensitive to methioninemediated repression.
\end{abstract}

Keywords: Aspergillus nidulans, folate regulatory genes

\section{INTRODUCTION}

Folates are essential cellular components serving as onecarbon units in the biosynthesis of methionine, thymidylate, the formyl group of fMet-tRNA in prokaryotes and interconversion of glycine and serine. There are several enzymes involved in folate metabolism, the core part of which is shown in Fig. 1. The regulation of the activity of these enzymes has been studied in various organisms (for reviews see McGuire \& Coward, 1984; Blakley, 1984; Rowe, 1984; Santi \& Danenberg, 1984; Schirch, 1984; Schirch \& Strong, 1989 and references therein). Much less is known about regulation of their synthesis, particularly about regulatory genes which control these processes. Two regulatory genes, met $J$ and $m e t R$, have been described in Escherichia coli and Salmonella typhimurium. The former is a negative regulator of a number of methionine biosynthetic genes including met $E$, encoding the vitamin $\mathrm{B}_{12}$-independent methyltransferase (for a review see Saint-Girons et al., 1988 ). The met $R$ gene product mediates the activation of the metE gene by homocysteine (Urbanowski \& Stauffer, 1989; Byerly et al., 1990) and also regulates expression of the glyA gene (Plamann \& Stauffer, 1989).

We have found that the synthesis of folate-dependent enzymes in Aspergillus nidulans is regulated by intracellular pools of homocysteine and methionine, the

Abbreviations: DHFR, dihydrofolate reductase; FPGS, folylpolyglutamate synthetase; MS, methionine synthase; MTFR, methylenetetrahydrofolate reductase; SHMT, serine hydroxymethyltransferase. former acting as an inducer and the latter as a corepressor (Nadolska-Lutyk et al., 1989; Natorff et al., 1993). This regulation is impaired by two, apparently allelic, mutations met $\mathrm{H} 2$ and metD10 (no recombinants among 4000 progeny in the cross met $\mathrm{H} 2 \times$ metD 10 ; Clutterbuck, 1987). Both mutants are leaky: the growth of metH2 is stimulated only by methionine, while

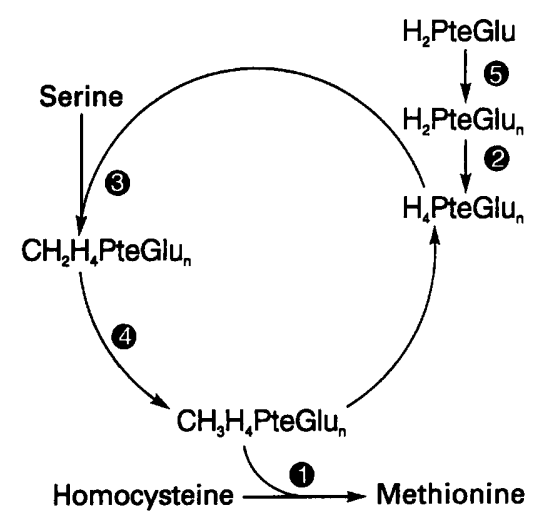

Fig. 1. An outline of folate metabolism.1, Methionine synthase (MS); 2, dihydrofolate reductase (DHFR); 3, serine hydroxymethyltransferase (SHMT); 4, methylenetetrahydrofolate reductase (MTFR); 5, folylpolyglutamate synthetase (FPGS). $\mathrm{H}_{2}$ PteGlu $\mathrm{n}_{n}$ dihydropteroylpolyglutamate; $\mathrm{H}_{4}$ PteGlu $_{n}$, tetrahydropteroylpolyglutamate; $\mathrm{CH}_{2} \mathrm{H}_{4} \mathrm{PteGlu}_{n}$, methylenetetrahydropteroylpolyglutamate; $\mathrm{H}_{3} \mathrm{H}_{4}$ PteGlu $_{n}$, methyltetrahydropteroylpolyglutamate. 
metD10 also grows on betaine. The mutants show a repressed level of folylpolyglutamate synthetase (FPGS) coinciding with a ratio of folate monoglutamate to folate polyglutamates higher than that found in the wild-type (Lewandowska et al., 1993, 1996).

By searching for metH2 revertants we have isolated six methionine-independent strains, some of which show regulation of folate enzymes insensitive to methioninemediated repression (Lewandowska et al., 1996). In the present study we have characterized these strains. In three cases reversion occurred intragenically, while in the remaining three strains suppressor mutations occurred in independent but loosely linked loci, designated folA, folB and folC.

\section{METHODS}

Strains. The following strains of $A$. nidulans from our collection which carry standard markers (Clutterbuck, 1994) were used: $m e t H 2$, pyroA4, yA1; $\arg A 1, b i A 1$, pabaA2 and pyroA4, $y A 1$. The latter strain was used as a reference wildtype strain for enzyme assays. The met $\mathrm{H} 2$ revertants, R5, R7, $\mathrm{R} 8, \mathrm{R} 10, \mathrm{R} 14$ and R15, which in primary tests showed altered regulation of two folate-dependent enzymes as compared with the metH2 strain (Lewandowska et al., 1996), all carried the pyro $A 4$ and $y A 1$ markers. The tester strain FGSC 465 carrying the chromosome markers sulA1, acrA1, galA1, pyroA4, facA303, lacA1, choA1 and chaA1 was used for allocation of newly described genes in chromosomes.

Media, culture conditions and enzyme assays. The strains were grown in liquid minimal medium (Paszewski \& Grabski, 1974) which was supplemented with $5 \mathrm{mM}$ L-methionine as indicated, in a rotary shaker $\left(200\right.$ r.p.m.) at $30^{\circ} \mathrm{C}$ for $18 \mathrm{~h}$. Mycelia were collected on a glass filter, washed thoroughly with water and blotted on filter paper. Cell-free extracts for enzyme assays were prepared by mixing mycelial pads with equal volumes of powdered glass and grinding in a chilled mortar with $0.1 \mathrm{M}$ potassium phosphate buffer $(\mathrm{pH} 7 \cdot 5$ ). The resulting slurry was centrifuged at $10000 \mathrm{~g}$ for $15 \mathrm{~min}$ at $4^{\circ} \mathrm{C}$ and the supernatant used as an enzyme source. Freezing of the extracts did not affect the activity of the enzymes studied.

Folylpolyglutamate synthetase (FPGS) (EC 6.3.2.17) was assayed according to McGuire et al. (1980), methionine synthase (MS) (5-methyltetrahydrofolate: L-homocysteine methyltransferase; EC 2.1.1.13) was assayed as described previously (Paszewski et al., 1977), serine hydroxymethyltransferase (SHMT) (EC 2.1.2.1) and methylenetetrahydrofolate reductase (MTFR) (EC 1.5.1.20) were assayed according to Mudd et al. (1972), and dihydrofolate reductase (DHFR) (EC 1.5 .1.3) was assayed as described by Hanggi \& Littlefield (1974). Protein was estimated according to Bradford (1976) with bovine serum albumin (fraction V) as a standard.

Isolation and genetic analysis of metH2 suppressors. Conidia of the metH2, pyroA4, yA1 strain suspended in water $\left(10^{8}\right.$ conidia $\mathrm{ml}^{-1}$ ) were irradiated by UV light (survival about $1 \%$ ) and plated on minimal medium. Methionine-independent colonies were isolated and analysed genetically and biochemically. Genetic crosses followed the procedures of Pontecorvo et al. (1953). Genes were allocated to their linkage groups by mitotic haploidization (Forbes, 1959) facilitated by the use of benlate (Hastie, 1970).

\section{RESULTS}

\section{Isolation of metH2 suppressor mutations and their genetic analysis}

About $50 \mathrm{Met}^{+}$colonies were obtained after mutagenesis of the metH2, pyroA4, yA1 strain. Six of these, R5, R7, $\mathrm{R} 8, \mathrm{R} 10, \mathrm{R} 14$ and R15, representing different phenotypes (Fig. 2), were chosen at random. They were crossed with the wild-type. In the case of R5, R7 and R14 strains no methionine-requiring segregants were recovered among 125-150 progeny scored. These three strains grow more slowly than the wild-type on minimal medium. Their phenotypes suggest that reversion resulted from intragenic suppressors or mutations in an adjacent gene rather than from back mutations. These suppressor mutations are designated metH2R5, metH2R7 and metH2R14. They are all dominant in heterokaryons with the metH2 strain. Crosses of the R8, R10 and R15 strains with the wild-type gave about $25 \% \mathrm{Met}^{-}$ segregants, indicating that reversion resulted from a suppressor mutation in an independent gene. From each cross a few $\mathrm{Met}^{+}$segregants were chosen for enzymic examination. Those which showed deregulation of folate-dependent enzymes were crossed with the wild-type strain to select segregants carrying only a suppressor mutation, i.e. not segregating the metH2 mutation, and segregants carrying both mutations but with different auxotrophic markers. The latter strains were crossed pairwise to find out how many genes represent the suppressor mutations. The results indicated that the three suppressor mutations studied occurred in different but linked genes. These genes, which we designate folA, folB and folC, are mutated in the R8, R10 and R15 strains, respectively.

The genes are arranged in the order folB-folA-folC, with $17 \cdot 2 \%$ linkage between folB and folA, and $20.4 \%$ linkage between folA and folC.

The linkage values are based on 381 and 581 segregants scored in the crosses folA 8 metH $2 \times$ folB10 metH 2 and folA8 met $\mathrm{H} 2 \times$ folC15 metH2, respectively. In the cross folB10 metH2 $\times$ folC15 metH2 $117 \mathrm{Met}^{-}$colonies were found among 428 tested, which gives a recombination frequency of $54.6 \%$. The fol genes were allocated to the linkage group VI by mitotic haploidization with the use of the tester strain FGSC 465 and, additionally, with a strain carrying the $\operatorname{argA1}$ marker located in the same chromosome. As the suppressor phenotype is not always easily distinguishable we determined chromosome segregation in haploids carrying both metH2 and the suppressor mutation. The folA 8 and folC15 mutations are recessive, while folB10 is semidominant, as determined in heterokaryons between the original suppressor strains and the metH2 mutant.

\section{Regulation of folate-dependent enzymes in metH2 suppressor strains}

The results presented in Table 1 indicate several marked differences in enzyme levels between strains carrying extragenic suppressors and the met $\mathrm{H} 2$ and wild-type 
(a)

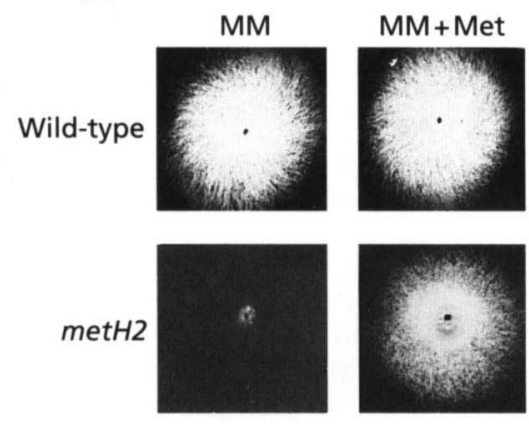

(c)

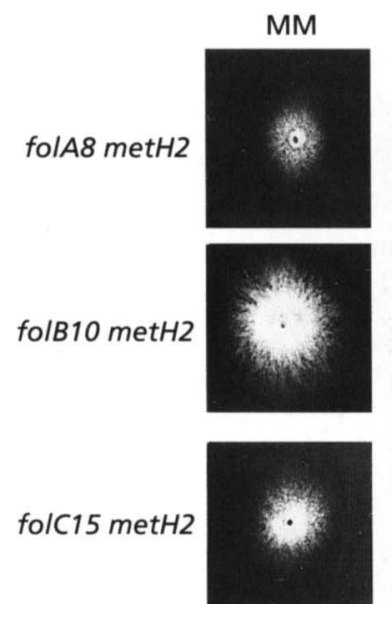

(b)
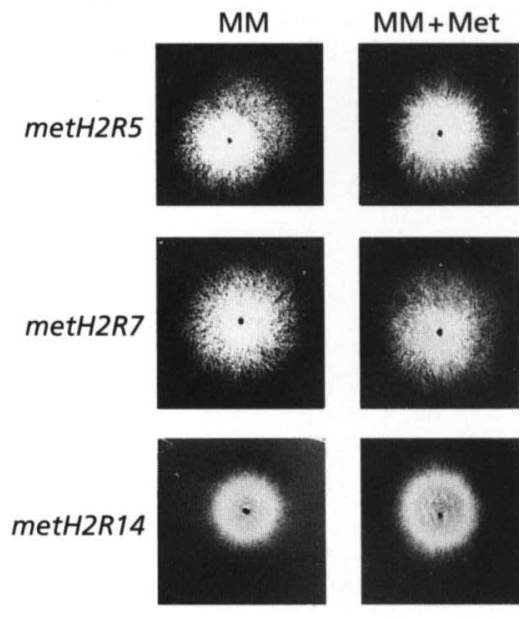

(d)
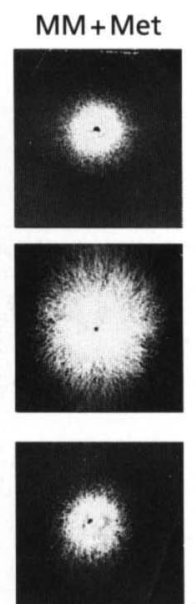
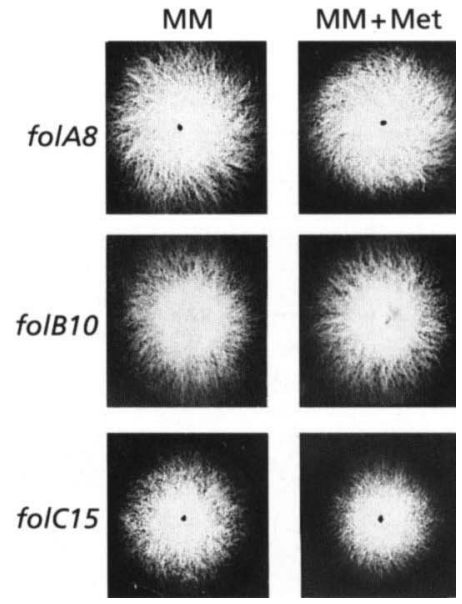

Fig. 2. (a) Growth of the metH2 (pyroA4, yA1) and wild-type (pyroA4, yA1) strains; (b) growth of strains carrying metH2 intragenic suppressors: metH2R5 (pyroA4, yA1), metH2R7 (pyroA4, yA1), metH2R14 (pyroA4, yA1); (c) growth of strains carrying fol mutations: folA8 metH2 (pyroA4, yA1), folA8 (pyroA4, yA1), folB10 metH2 (pyroA4, yA1), folB10 (pyroA4, yA1), folC15 metH2 (pyroA4, yA1) and folC15 (yA1). In each case growth was for $48 \mathrm{~h}$ at $37^{\circ} \mathrm{C}$. MM, minimal medium; $\mathrm{MM}+$ Met, minimal medium supplemented with $5 \mathrm{mM}$ L-methionine.

strains. These differences are particularly pronounced in the case of FPGS. The mutations folA8, folB10 and folC15 result in an elevated level of this enzyme and, what is more important, relieve its synthesis, completely or partially, from methionine-mediated repression. Essentially, the same is true in the case of MS and DHFR. MTFR is elevated in all strains carrying fol mutations but in the case of folB10 metH2 and folB10 strains grown in the presence of methionine a repressed enzyme level is observed. The SHMT levels in the revertants do not appear to differ from that of the wild-type strain except for the folA8 metH2 and folC15 metH2 strains where the enzyme appears to be less repressed in the presence of methionine.

The results shown in Table 1 also indicate that the mode of folate enzyme regulation in the fol mutants is essentially similar to that observed in the fol met $\mathrm{H} 2$ strains, with the exception of strains carrying the folA8 mutation: the folA8/17 strain shows higher levels of MS, MTFR and DHFR. Interestingly, the two segregants from the cross folA 8 met $\mathrm{H} 2 \times$ wild-type carrying the folA mutation, folA8/9 and folA8/17, differ in the level of MS in minimal-grown cells, indicating that genetic background may modulate the folA action.

The folate-dependent enzyme levels in the strains carrying intragenic suppressors are shown in Table 2. In all three strains grown in minimal medium the FPGS level is slightly higher than in the wild-type but the enzyme is repressible in the metH2R5 and metH2R7 strains but not in the metH2R14 strain. On the other hand MS is not repressible in the metH2R7 and met $H 2 R 14$ strains. In all three strains grown in minimal medium the MTFR and DHFR levels are elevated as compared to the wild-type but repressed in the presence 
Table 1. Activity of folate-dependent enzymes in the wild-type, metH2 and fol strains of $A$. nidulans

Results represent mean values $\pm \mathrm{SD}$ from three or more experiments. In a few cases two experiments were performed and in three cases only one. Enzyme activities exceeding the corresponding wild-type values by more than $50 \%$ are shown in bold type. MM, minimal medium; + Met, minimal medium supplemented with $5 \mathrm{mM}$ L-methionine.

\begin{tabular}{|c|c|c|c|c|c|c|c|c|c|c|}
\hline \multirow[t]{3}{*}{ Strain } & \multicolumn{10}{|c|}{ Specific activity $\left.\left[\mathrm{nmol} \mathrm{h} \mathrm{h}^{-1} \text { (mg protein }\right)^{-1}\right]$ of: } \\
\hline & \multicolumn{2}{|c|}{ FPGS } & \multicolumn{2}{|c|}{ MS } & \multicolumn{2}{|c|}{ MTFR } & \multicolumn{2}{|c|}{ DHFR } & \multicolumn{2}{|c|}{ SHMT } \\
\hline & $\mathbf{M M}$ & + Met & $\mathbf{M M}$ & + Met & MM & + Met & $\mathbf{M M}$ & + Met & $\mathbf{M M}$ & + Met \\
\hline Wild-type & $4 \cdot 56 \pm 0 \cdot 26$ & $0.76 \pm 0.06$ & $12 \cdot 70 \pm 0.14$ & $6 \cdot 50 \pm 0 \cdot 14$ & $2 \cdot 72 \pm 0 \cdot 10$ & $0.86 \pm 0.05$ & $17 \cdot 48 \pm 0.97$ & $8.07 \pm 1 \cdot 40$ & $4266 \pm 417$ & $2236 \pm 70$ \\
\hline met $H 2$ & $1 \cdot 29 \pm 0.00$ & $0.73 \pm 0.00$ & $10 \cdot 00 \pm 2 \cdot 00$ & $5.90 \pm 0.20$ & $3 \cdot 00 \pm 0 \cdot 20$ & $1 \cdot 20 \pm 0 \cdot 20$ & $20 \cdot 50 \pm 0 \cdot 50$ & $9 \cdot 50 \pm 0 \cdot 70$ & $4666 \pm 152$ & $2826 \pm 253$ \\
\hline $\begin{array}{l}\text { folA8 } \\
\qquad \text { met } H 2\end{array}$ & $8 \cdot 76 \pm 3 \cdot 50$ & $4.71 \pm 0.06$ & $15 \cdot 45 \pm 1 \cdot 90$ & $12 \cdot 85 \pm 0.07$ & $3 \cdot 23 \pm 0 \cdot 15$ & $1 \cdot 03 \pm 0.20$ & $21 \cdot 56 \pm 0.25$ & $9 \cdot 06 \pm 1.32$ & $3686 \pm 57$ & $3092 \pm 70$ \\
\hline folA $8 / 9$ & $9 \cdot 80$ & $4.35 \pm 0.35$ & $14.00 \pm 0.00$ & $8.40 \pm 0.00$ & $3 \cdot 60 \pm 0 \cdot 34$ & $2.00 \pm 0.74$ & $22 \cdot 90 \pm 0.85$ & $12.80 \pm 0.91$ & $3891 \pm 71$ & $3081 \pm 85$ \\
\hline folA $8 / 17$ & $7.75 \pm 2.37$ & $3 \cdot 55 \pm 1 \cdot 18$ & $22.70 \pm 0.42$ & $17 \cdot 00 \pm 6 \cdot 20$ & $3.80 \pm 0.00$ & $3 \cdot 26 \pm 0 \cdot 30$ & $24.46 \pm 0.56$ & $21 \cdot 40 \pm 4 \cdot 65$ & $4406 \pm 7$ & $2771 \pm 56$ \\
\hline $\begin{array}{l}\text { folB10 } \\
\quad \text { metH2 }\end{array}$ & $5 \cdot 80 \pm 0 \cdot 75$ & $2 \cdot 70 \pm 1 \cdot 80$ & $17 \cdot 76 \pm 1 \cdot 09$ & $8 \cdot 70 \pm 2 \cdot 30$ & $3 \cdot 86 \pm 0.45$ & $0.93 \pm 0.32$ & $22 \cdot 35 \pm 0.68$ & $12 \cdot 50 \pm 6 \cdot 10$ & $3725 \pm 97$ & $2371 \pm 338$ \\
\hline folB10 & $5 \cdot 30$ & 1.50 & $17 \cdot 10 \pm 1 \cdot 83$ & $6.45 \pm 0.35$ & $4.95 \pm 0.80$ & $0.76 \pm 0.05$ & $25 \cdot 46 \pm 1 \cdot 18$ & $8 \cdot 10 \pm 1 \cdot 70$ & $4228 \pm 130$ & $2056 \pm 22$ \\
\hline $\begin{array}{l}\text { folC15 } \\
\text { metH2 }\end{array}$ & $7 \cdot 80 \pm 4 \cdot 20$ & $5 \cdot 35 \pm 0.07$ & $21 \cdot 25 \pm 1 \cdot 60$ & 16.90 & $5 \cdot 40 \pm 2 \cdot 12$ & $3.20 \pm 0.56$ & $28 \cdot 05 \pm 4 \cdot 10$ & $19 \cdot 80 \pm 2 \cdot 77$ & $4489 \pm 527$ & $3126 \pm 22$ \\
\hline folC15 & $12 \cdot 87 \pm 0 \cdot 15$ & $6.40 \pm 0.46$ & $19.60 \pm 0.70$ & $17.80 \pm 0.84$ & $6 \cdot 72 \pm 0.23$ & $3 \cdot 60 \pm 0 \cdot 16$ & $31 \cdot 40 \pm 4 \cdot 40$ & $20 \cdot 64 \pm 1.20$ & $4289 \pm 68$ & $3214 \pm 28$ \\
\hline
\end{tabular}

\section{Table 2. Activity of folate-dependent enzymes in the wild-type and metH2R strains of Aspergillus nidulans}

Results represent mean values $\pm S D$ from three or more experiments. In a few cases only two experiments were performed. Enzyme activities exceeding the corresponding values for the wild-type strain by more than $50 \%$ are shown in bold type. MM, minimal medium; + Met, minimal medium supplemented with $5 \mathrm{mM}$ L-methionine.

\begin{tabular}{|c|c|c|c|c|c|c|c|c|c|c|}
\hline \multirow[t]{3}{*}{ Strain } & \multicolumn{10}{|c|}{ Specific activity $\left[\mathrm{nmol} \mathrm{h} \mathrm{h}^{-1}(\mathrm{mg} \text { protein })^{-1}\right]$ of: } \\
\hline & \multicolumn{2}{|c|}{ FPGS } & \multicolumn{2}{|c|}{ MS } & \multicolumn{2}{|c|}{ MTFR } & \multicolumn{2}{|c|}{ DHFR } & \multicolumn{2}{|c|}{ SHMT } \\
\hline & MM & + Met & $\mathbf{M M}$ & + Met & $\mathbf{M M}$ & + Met & $\mathbf{M M}$ & + Met & MM & + Met \\
\hline Wild-type & $4 \cdot 56 \pm 0 \cdot 26$ & $0.76 \pm 0.06$ & $12 \cdot 70 \pm 0 \cdot 14$ & $6 \cdot 50 \pm 0 \cdot 14$ & $2 \cdot 72 \pm 0 \cdot 10$ & $0.86 \pm 0.05$ & $17 \cdot 48 \pm 0.97$ & $8.07 \pm 1.40$ & $4266 \pm 417$ & $2236 \pm 70$ \\
\hline metH2 & $1 \cdot 29 \pm 0.00$ & $0.73 \pm 0.00$ & $10 \cdot 00 \pm 2 \cdot 00$ & $5 \cdot 90 \pm 0 \cdot 20$ & $3 \cdot 00 \pm 0 \cdot 20$ & $1 \cdot 20 \pm 0 \cdot 20$ & $20 \cdot 50 \pm 0.50$ & $9 \cdot 50 \pm 0 \cdot 70$ & $4666 \pm 152$ & $2826 \pm 253$ \\
\hline metH2R5 & $4 \cdot 80 \pm 0.48$ & $0 \cdot 89 \pm 0.19$ & $14 \cdot 46 \pm 0 \cdot 46$ & $7 \cdot 50 \pm 0 \cdot 14$ & $3.60 \pm 0.00$ & $0.80 \pm 0.00$ & $18.65 \pm 0.77$ & $5 \cdot 75 \pm 0 \cdot 90$ & $2625 \pm 267$ & $1687 \pm 63$ \\
\hline $\operatorname{met} H 2 R 7$ & $5.38 \pm 0.32$ & $1 \cdot 16 \pm 0.27$ & $17 \cdot 46 \pm 1 \cdot 16$ & $17 \cdot 10 \pm 0 \cdot 00$ & $3.70 \pm 0.14$ & $0.65 \pm 0.07$ & $20.75 \pm 0.91$ & $8.40 \pm 0.28$ & $2814 \pm 000$ & $1626 \pm 000$ \\
\hline metH2R14 & $5.46 \pm 0.69$ & $5.46 \pm 0.28$ & $13 \cdot 80 \pm 1 \cdot 17$ & $12.75 \pm 0.20$ & $4.00 \pm 0.28$ & $1.50 \pm 0.14$ & $22.35 \pm 0.77$ & $13 \cdot 10 \pm 1 \cdot 27$ & $2686 \pm 000$ & $1558 \pm 113$ \\
\hline
\end{tabular}

of methionine. However, the repression is markedly weaker in the metH2R14 strain. Interestingly all three strains exhibit an SHMT level lower than that found in the wild-type and met $\mathrm{H} 2$ strains. This level is even lower when methionine is present in the growth medium.

\section{DISCUSSION}

We demonstrated in our earlier studies that the synthesis of a number of folate-dependent enzymes in A. nidulans is regulated by a system in which homocysteine acts as an inducer and methionine as a co-repressor (Lewandowska et al., 1993, 1996). This regulatory system is impaired in the metH2 and metD10 mutants, which is particularly manifested by a repressed (or non-inducible) level of FPGS.

By isolating metH2 revertants we selected six strains in which synthesis of folate-dependent enzymes was in- sensitive, to differing extents, to methionine-mediated repression. In three of these strains reversion was due, most likely, to an intragenic suppressor mutation. Clearly, these mutations differ in their effect on folate enzyme synthesis. For instance FPGS and MS are not repressible in the metH2R14 strain while in the metH2R7 strain only the latter enzyme is. It is worth noting that the SHMT level in these strains is lower than that in the wild-type and metH2 strain. If these variations in the regulatory effects occur at the transcriptional level they might reflect differences in the responsive regulatory elements in the promoters of the structural genes encoding particular enzymes. A similar reasoning may be applied to explain differences in effects of suppressor mutations occurring in three independent genes, designated fol $A$, folB and folC. We assume as the simplest hypothesis that these are regulatory genes which control synthesis of folate enzymes although 
there is no direct evidence that their primary effect is regulatory.

Expression of particular structural genes may be regulated by different transcriptional complexes which form at various promoters. The fol gene products altered by mutation might affect the properties of these complexes. The different protein sequences resulting from the various alleles of these genes might have specific and unpredictable effects on the modulation of regulation.

To our knowledge the fol genes described here, together with the metH/metD locus, are the first identified eukaryotic regulatory genes controlling folate metabolism, although the existence of such genes can plausibly be deduced from results obtained with other organisms. For instance a three- to fourfold increase in DHFR and thymidylate synthase transcripts observed in human cisplatin-resistant cells (Scanlon \& Kashani-Sabet, 1988) can be attributed to a modulation in activity of a regulatory gene.

It appears that the system governing the regulation of folate-dependent enzymes in A. nidulans may be rather complex, especially taking into account the possibility that the list of genes involved may not be complete yet. This conclusion is supported by the fact that mutants representing three different loci were identified among only six mutants tested from 50 originally isolated. It is very likely that among the enzymes studied here DHFR is also regulated by cell-cycle-controlling genes. The complexity of mammalian DHFR gene promoter structure as well as the transcriptional complex involving both nonspecific and specific proteins (for review see Slansky \& Farnham, 1996) is compatible with the idea of a multigene regulatory system that might control the expression of a particular structural gene. In this connection it is worth noting that genes encoding SHMT in Neurospora crassa (McClung et al., 1992) and Saccharomyces cerevisiae (McNeil et al., 1994) are under control of the amino acid cross-pathway regulatory system.

In our previous work (Natorff et al., 1993) we found that the scon mutations, which impair the sulphur metabolite repression system, also lead to the derepression of a number of folate-dependent enzymes. It was shown that this derepression is a secondary effect - it results from an increased pool of homocysteine. The direct role of the scon gene products is the regulation of sulphurrelated genes. It will be interesting, in turn, to check if the fol genes are specific for folate metabolism, or also affect, directly or indirectly, sulphur metabolism.

\section{ACKNOWLEDGEMENTS}

This work was supported by the Polish State Committee for Scientific Research, grant no. 6P 20300407.

\section{REFERENCES}

Blakley, R. L. (1984). Dihydrofolate reductase. In Folates and Pterins, pp. 191-253. Edited by R. L. Blakely \& S. J. Bencovic. New York: Wiley.
Bradford, M. M. (1976). A rapid and sensitive method for the quantitation of microgram quantities of protein utilizing the principle of protein-dye binding. Anal Biochem 72, 248-254.

Byerly, K. A., Urbanowski, M. L. \& Stauffer, G. V. (1990). Escherichia coli met $R$ mutants that produce a metR activator protein with an altered homocysteine response. J Bacteriol 172, 28392843.

Clutterbuck, A. J. (1987). Revised allelism relationships among Aspergillus nidulans met and gal mutants. Fungal Genet Newsl 34, 27.

Clutterbuck, A. J. (1994). Linkage map and loci. In Aspergillus : 50 Years On, pp. 791-823. Edited by S. D. Martinelli \& J. R. Kinghorn. Amsterdam: Elsevier.

Forbes, E. (1959). Use of mitotic segregation for assigning genes to linkage groups in Aspergillus nidulans. Heredity 13, 67-80.

Hanggi, V. J. \& Littlefield, J. W. (1974). Isolation and characterization of the multiple forms of dihydrofolate reductase from methotrexate resistant hamster cells. J Biol Chem 249, 1390-1397.

Hastie, A. C. (1970). Benlate-induced instability of Aspergillus diploids. Nature 226, 771.

Lewandowska, I., Sikora, E., Szablewska, I., Balińska, M. \& Paszewski, A. (1993). Metabolism of folate glutamates in Aspergillus nidulans. In Chemistry and Biology of Pteridines and Folates, pp. 675-677. Edited by J. E. Ayling, M. G. Nair \& C. M. Baugh. New York: Plenum.

Lewandowska, I., Baliniska, M., Natorff, R. \& Paszewski, A. (1996). Regulation of folate-dependent enzyme levels in Aspergillus nidulans: studies with regulatory mutants. Biochim Biophys Acta 1290, 89-94.

McClung, C. R., Davis, C. R., Page, K. M. \& Denome, S. A. (1992). Characterization of the formate (for) locus, which encodes the cytosolic serine hydroxymethylthransferase of Neurospora crassa. Mol Cell Biol 12, 1412-1421.

McGuire, J. J. \& Coward, J. K. (1984). Pteroylpolyglutamates: biosynthesis, degradation and function. In Folates and Pterins, pp. 135-190. Edited by R. L. Blakely \& S. J. Bencovic. New York: Wiley.

McGuire, J. J., Hsich, P., Coward, J. K. \& Bertino, J. R. (1980). Enzymatic synthesis of folylpolyglutamates. J Biol Chem 255, 5776-5788.

McNeil, J. B., McIntosh, E. M., Taylor, B. V., Zhang, F., Tang, S. \& Bognar, A. L. (1994). Cloning and molecular characterization of three genes, including two genes encoding serine hydroxymethyltransferases, whose inactivation is required to render yeast auxotrophic for glycine. J Biol Chem 269, 9155-9165.

Mudd, S. H., Uhlendorf, B. W., Freeman, J. M., Finkelstein, J. D. \& Shih, V. E. (1972). Homocystinuria associated with decreased methylenetetrahydrofolate reductase activity. Biochem Biophys Res Commun 46, 905-912.

Nadolska-Lutyk, J., Balińska, M. \& Paszewski, A. (1989). Interrelated regulation of sulphur-containing amino acid biosynthetic enzymes and folate-metabolizing enzymes in Aspergillus nidulans. Eur J Biochem 181, 231-235.

Natorff, R., Balinska, M. \& Paszewski, A. (1993). At least four regulatory genes control sulphur metabolite repression in Aspergillus nidulans. Mol Gen Genet 238, 185-192.

Paszewski, A. \& Grabski, J. (1974). Regulation of S-amino acids biosynthesis in Aspergillus nidulans. Mol Gen Genet 132, 307-320.

Paszewski, A., Prazmo, W. \& Landman-Balinska, M. (1977). Regulation of homocysteine metabolizing enzymes in Aspergillus nidulans. Mol Gen Genet 155, 109-112. 
Plamann, M. D. \& Stauffer, G. V. (1989). Regulation of the Escherichia coli glyA gene by the metR gene product and homocysteine. J Bacteriol 171, 4958-4962.

Pontecorvo, G., Roper, J. A., Hemmons, L. M., Macdonald, K. D. \& Bufton, A. W. (1953). The genetics of Aspergillus nidulans. Adv Genet 5, 141-238.

Rowe, P. B. (1984). Folates in the biosynthesis and degradation of purines. In Folates and Pterins, pp. 329-344. Edited by R. L. Blakely \& S. J. Bencovic. New York: Wiley.

Saint-Girons, T., Parsot, C., Zakin, M. M., Barzu, O. \& Cohen, G. N. (1988). Methionine biosynthesis in Enterobacteriaceae: biochemical, regulatory and evolutionary aspects. Crit Rev Biochem 23, S1-S42.

Santi, D. V. \& Denenberg, P. V. (1984). Folates in pyrimidine nucleotide biosynthesis. In Folates and Pterins, pp. 345-398. Edited by R. L. Blakely \& S. J. Bencovic. New York: Wiley.

Scanlon, K. J. \& Kashani-Sabet, M. (1988). Elevated expression of thymidylate synthase cycle genes in cisplatin-resistant human ovarian carcinoma A2780 cells. Proc Natl Acad Sci USA 85, 650-653.

Schirch, V. (1984). Folates in serine and glycine metabolism. In Folates and Pterins, pp. 399-431. Edited by R. L. Blakely \& S. J. Bencovic. New York: Wiley.

Schirch, V. \& Strong, W. B. (1989). Interaction of folylpolyglutamates with enzymes in one-carbon metabolism. Arch Biochem Biophys 269, 371-380.

Slansky, J. E. \& Farnham, P. J. (1996). Transcriptional regulation of the dihydrofolate reductase gene. BioEssays 18, 55-62.

Urbanowski, M. L. \& Stauffer, G. V. (1989). Role of homocysteine in $m e t R$-mediated activation of the $m e t E$ and $m e t H$ genes in Salmonella typhimurium and Escherichia coli. J Bacteriol 171, 3277-3281.

Received 21 April 1997; revised 17 June 1997; accepted 25 June 1997. 